\title{
Delays in Initiation of Disease-Modifying Therapy in Rheumatoid Arthritis Patients: Data from a US-Based Registry
}

Dimitrios A. Pappas · Jeffrey D. Kent · Jeffrey D. Greenberg •

Marc A. Mason · Joel M. Kremer · Robert J. Holt

Received: June 17, 2015 / Published online: September 29, 2015

(C) The Author(s) 2015. This article is published with open access at Springerlink.com

\section{ABSTRACT}

Introduction: The goal of this study was to evaluate how frequently rheumatoid arthritis (RA) therapy is instituted promptly and to describe the characteristics of patients who are not treated early upon diagnosis.

Electronic supplementary material The online version of this article (doi:10.1007/s40744-015-0019-6) contains supplementary material, which is available to authorized users.

D. A. Pappas $(\bowtie)$

Columbia University, New York, NY, USA

e-mail: dpappas@corrona.org;

dp2544@columbia.edu

D. A. Pappas · J. D. Greenberg · M. A. Mason

Corrona LLC, Southborough, MA, USA

J. D. Kent

Horizon Pharma USA, Inc., Deerfield, IL, USA

J. D. Greenberg

NYU School of Medicine, New York, NY, USA

M. A. Mason

University of Delaware, Newark, DE, USA

J. M. Kremer

Albany Medical College and The Center for

Rheumatology, Albany, NY, USA

R. J. Holt

University of Illinois-Chicago, Chicago, IL, USA
Methods: The percentage of patients who at the time of enrollment in the Corrona registry were not receiving any RA-directed therapy was evaluated and their characteristics were summarized. The time to subsequent initiation of any RA-directed therapy was also estimated.

Results: Among 35,485 patients enrolled in Corrona, 34,735 (97.9\%) were on appropriate therapy for RA and 750 (2.1\%) had no history of any RA-directed therapy at time of enrollment. Among patients without any history of RA-directed therapy, the overall disease duration was $5.5 \pm 9.0$ years, with only $50.7 \%$ of patients having early disease (duration $\leq 1$ year). Patients with no history of directed RA therapy did not have lower disease activity at enrollment compared with those receiving therapy. Clinical Disease Activity Index (CDAI) was $18.3 \pm 15.0 ; 34 \%$ of patients had high and $27.6 \%$ moderate disease activity by CDAI. Patients were followed for a median (95\% CI) time of 29.5 months (24.6-33.8). During the follow-up period, 372 out of 750 (49.6\%) patients initiated RA-directed therapy. The median time to initiation of any RA-directed therapy was 12.1 months (95\% CI 9.3-14.8). 
Conclusion: In this registry analysis, approximately $98 \%$ of patients were on appropriate RA therapy for their RA. However, a minority of patients with RA did not have a history of receiving disease-modifying therapy within a mean of approximately 5 years of RA onset and approximately $50 \%$ of them did not initiate any therapy within 12 months of registry follow-up. This delay in therapy did not appear to be related to a better controlled, or lower, RA disease activity state at the time of enrollment in the registry.

Funding: Corrona, LLC.

Keywords: Delayed therapy; Registry; Rheumatoid arthritis

\section{INTRODUCTION}

Early and aggressive therapy for rheumatoid arthritis (RA) with disease-modifying antirheumatic agents (DMARDs), glucocorticoids, and biologic agents is recommended by current treatment guidelines and supported by interventional studies with treat to target principles [1]. According to the current guidelines, diagnosis should be established as early as possible, therapy should be initiated immediately and escalated promptly to achieve low disease activity or remission. This goal should be reached within 3 to 6 months from diagnosis [1].

The consequences of delays in adequate therapy have been well documented in the literature and have driven the development of aggressive guidelines for the early treatment of RA [2-10]. Over the last several years, it has been repeatedly demonstrated that postponements in the aggressive, early treatment of joint inflammation result in deleterious effects on radiographic and other outcomes; joint damage and disability ensue, uncontrolled disease activity further undermines patients' quality of life, and uncontrolled inflammation increases the risk of comorbidities and accelerates mortality [1, 11]. Importantly, it has been proven that achieving a low disease activity outcome is more likely when there are no delays in initiation of therapy and that a "window of opportunity" exists early after the onset of disease during which RA is more susceptible to treatment [12].

Despite the above, several reports-mainly from European countries-show that only a minority of patients initiate disease-modifying therapy within the first few months of disease onset [13, 14]. Similar reports evaluating in detail the frequency, extent and causes of delaying initiation of RA therapy in the United States (US) are not available. Our goal in the present study was to evaluate how frequently RA therapy is not instituted promptly, to describe the characteristics of patients who are not treated early upon diagnosis, and to evaluate the time interval until initiation of therapy. The largest disease-based RA registry in the US was used to address the aforementioned questions.

\section{METHODS}

\section{Study Population}

Patients included in this analysis were subjects with RA enrolled in the Corrona registry, a disease-based, multicenter, observational registry, which enrolls and follows longitudinally adult patients with a diagnosis of RA according to the treating rheumatologist. The Corrona US RA registry has previously been 
extensively described elsewhere [15]. As of December 2014, data collected from approximately 40,200 patients during regular clinical encounters have yielded 291,672 patient visits and approximately 123,182 patient-years of follow-up observation time, with a mean patient follow-up time of 3.7 years (median 2.84 years).

A diverse geographic distribution of participating rheumatology practices is represented in the Corrona registry with 165 private and academic sites across 41 states in the US as of December 2014. All practices were evaluated prior to selection as to their experience with observational or interventional research. On-site and online training of personnel who participate in the registry with training on the mechanics of how to accurately and completely enter data into the case report forms occurred prior to participation. Corrona rheumatologists were instructed to enroll consecutive patients to maximize representativeness of the enrolled population.

Approvals for data collection and analyses of the Corrona RA registry were obtained from local institutional review boards of participating academic sites and a central institutional review board for private practice sites.

\section{Data Collection}

The methods of data collection in Corrona have been extensively described previously [15]. In brief, data are collected from both patients and physicians at enrollment and then at follow-up visits, which occur approximately every 4-6 months. The enrollment questionnaires capture general medical history including details about medications prescribed prior to enrollment in the registry. At follow-up visits, all the above data are updated; medication changes at and between visits are recorded, and disease activity at the time of the follow-up visit is captured.

All data are first captured on paper questionnaires and then entered in an electronic data capture system. A quality control process is in place to ensure the collection of robust, reliable and validated data. First, the electronic data capture system used has a built-in capacity to automatically identify erroneous or discrepant data entries and immediately indicates the need for corrections. Subsequently, a dedicated quality control team reviews the entered data on a regular basis and communicates directly with the participant rheumatology practice to correct possible mistakes in data entry.

\section{Statistical Analysis}

All Corrona patients enrolled in the registry as of March 31, 2013 were included in this analysis. Patients who had not received any disease-modifying therapy at the time of enrollment were identified and compared with patients with a history of RA-directed therapy at the time of enrollment in the registry. We followed the patients on no therapy longitudinally to calculate the time to initiation of any RA-directed therapy.

Descriptive statistics were examined at Corrona registry enrollment among the subgroups of patients with and without prior identified RA-directed therapy at time of enrollment. We used Student's $t$ tests and Chi-square tests to compare demographic and clinical characteristics between the two patient subgroups. To account for the presence of contraindications or comorbidities as a reason for delayed therapy, we assessed the comorbidity histories among the patient subgroups including history of malignancy, 
serious infections, cardiovascular disease, and hepatic disease.

We used the Kaplan-Meier method to calculate the median time to initiation of any drug used for the therapy of RA: prednisone, methotrexate (MTX), other conventional or targeted synthetic and biologic DMARDs within the subgroup of Corrona RA patients that had no identified RA-directed therapy at time of Corrona enrollment and who had not received any such therapy from the moment of diagnosis to the time of Corrona enrollment. These patients were followed longitudinally until March 2013.

STATA 13 (StataCorp LP, College Station, TX, USA) was used for all statistical analyses.

\section{RESULTS}

At the time of the analysis 34,485 patients had been enrolled in the Corrona registry. Among the entire registry population, 750 patients (2.1\%) had not received any targeted synthetic or biologic DMARD or steroid therapy (RA-directed therapy) from the time of RA diagnosis to the time of enrollment in the registry as shown in Fig. 1, while 34,736 (97.9\%) had a history of treatment with RA-directed therapy at the time of enrollment to Corrona.

\section{Demographic Characteristics}

As shown in Table 1, analysis of demographic, lifestyle, and anthropometric characteristics showed that patients who had not received any RA-directed therapy by the time of enrollment were more frequently smokers $(20.1 \%$ vs. $15.6 \%$, $P<0.0001)$ and less frequently college level educated $\quad(43.7 \% \quad$ vs. $55.1 \%, \quad P<0.0001)$ compared to patients exposed to therapy. Patients without history of RA-directed therapy were notably more frequently without medical insurance $(5.8 \%$ vs. $2.3 \%, P<0.0001)$. There were no differences in age or racial distribution between the two populations.

\section{Disease Characteristics}

The comparison of disease characteristics between patients without history of RA-directed therapy at the time of enrollment compared to those who had a history of such therapy is shown in Table 2. Patients without a history of RA-directed therapy had a shorter duration of RA disease $(5.5 \pm 9.0$ years vs. $9.0 \pm 9.7$ years). However, almost half of those patients had established disease with a duration $>1$ year. The mean disease activity as measured by Clinical Disease Activity Index (CDAI) among

Fig. 1 Analytic sample of Corrona RA patients by therapy history. $R A$ rheumatoid arthritis

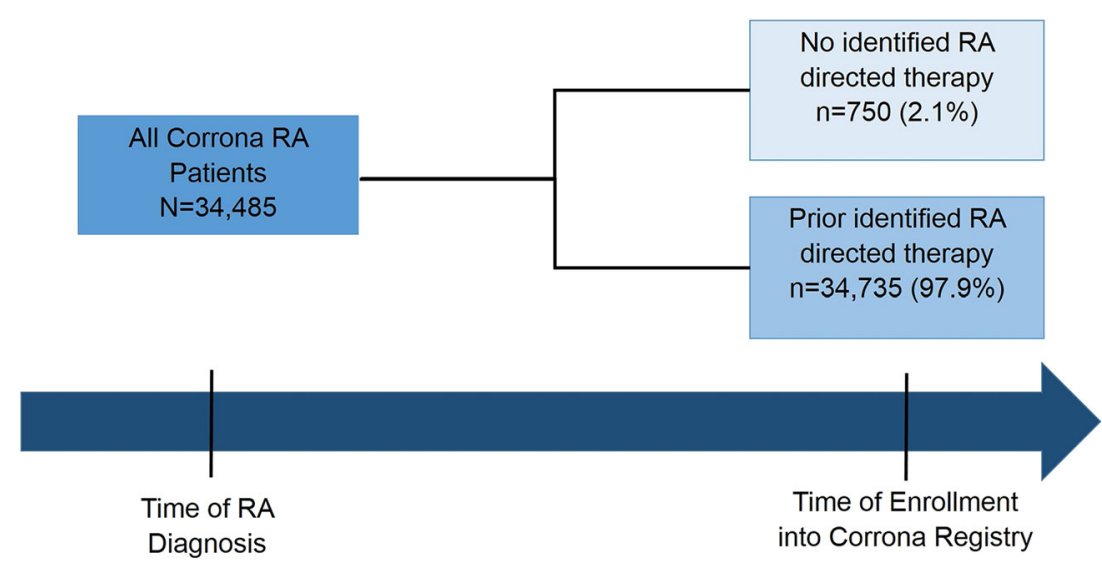


Table 1 Demographic characteristics of patients with RA by therapy history at time of enrollment into Corrona

\begin{tabular}{|c|c|c|c|}
\hline Characteristic & $\begin{array}{l}\text { No identified RA-directed therapy } \\
N=750 \\
n(\%)\end{array}$ & $\begin{array}{l}\text { Prior RA-directed therapy } \\
N=34,735 \\
n(\%)\end{array}$ & $P$ value ${ }^{a}$ \\
\hline Age (years), mean $\pm S D$ & $57.5 \pm 14.7$ & $58.1 \pm 13.7$ & 0.2104 \\
\hline Gender ( $\%$ female) & $567(75.9 \%)$ & $(76.40 \%)$ & 0.7520 \\
\hline White & $613(82.0 \%)$ & $(82.50 \%)$ & 0.7100 \\
\hline \multicolumn{4}{|l|}{ BMI } \\
\hline Mean \pm SD & $29.5 \pm 7.6$ & $29.3 \pm 7.2$ & 0.3761 \\
\hline Underweight/Normal & $215(30.5 \%)$ & $9896(29.5 \%)$ & 0.0320 \\
\hline Overweight & $198(28.1 \%)$ & $10,952(32.7 \%)$ & \\
\hline Obese & $291(41.3 \%)$ & $12,671(37.8 \%)$ & \\
\hline Education (\% college educated) & $304(43.7 \%)$ & $18,157(55.1 \%)$ & $<0.0001$ \\
\hline \multicolumn{4}{|l|}{ Smoking status } \\
\hline Never & $439(59.7 \%)$ & $20,415(59.3 \%)$ & $<0.0001$ \\
\hline Former & $149(20.2 \%)$ & $8662(25.2 \%)$ & \\
\hline Current & $148(20.1 \%)$ & $5353(15.6 \%)$ & \\
\hline Marital status (\% married) & $470(63.8 \%)$ & $22,110(64.5 \%)$ & 0.6970 \\
\hline \multicolumn{4}{|l|}{ Work status } \\
\hline Full time & $265(36.0 \%)$ & $12,885(37.3 \%)$ & 0.0010 \\
\hline Part time & $74(10.1 \%)$ & $3271(9.5 \%)$ & \\
\hline Disabled & $73(9.9 \%)$ & $4269(12.4 \%)$ & \\
\hline Retired & $188(25.5 \%)$ & $9419(27.3 \%)$ & \\
\hline Other $^{\mathrm{b}}$ & $136(18.5 \%)$ & $4676(13.6 \%)$ & \\
\hline \multicolumn{4}{|l|}{ Insurance $^{c}$} \\
\hline None & $34(5.8 \%)$ & $684(2.3 \%)$ & $<0.0001$ \\
\hline Private & $420(71.4 \%)$ & $21,822(73.7 \%)$ & 0.2580 \\
\hline Medicare & $154(26.2 \%)$ & $10,230(34.5 \%)$ & $<0.0001$ \\
\hline Medicaid & $55(9.9 \%)$ & $1909(6.4 \%)$ & 0.0040 \\
\hline
\end{tabular}

$B M I$ body mass index, $R A$ rheumatoid arthritis, $S D$ standard deviation

${ }^{a} P$ value from Student's two-sample two-sided $t$ test (continuous variables) and Chi-squared test (categorical variables) comparing patients having no identified RA-directed therapy at time of enrollment to those with RA-directed therapies

b "Other" includes "at home" and "student" categories

c Sum may not add up to total as some patients have more than one type of insurance

patients on no therapy was higher compared to patients on therapy $(18.3 \pm 15.0$ vs. $14.3 \pm 13.1)$. Last, the percentage of patients without therapy but with moderate and high disease activity was higher $(>60 \%)$ compared to those with prior therapy $(51.3 \%)$. In addition, morning stiffness 
Table 2 Disease characteristics of patients with RA by therapy history at time of enrollment into Corrona

\begin{tabular}{|c|c|c|c|}
\hline Characteristic & $\begin{array}{l}\text { No identified RA-directed therapy } \\
N=750 \\
n(\%)\end{array}$ & $\begin{array}{l}\text { Prior RA-directed therapy } \\
N=34,735 \\
n(\%)\end{array}$ & $P$ value ${ }^{\mathrm{a}}$ \\
\hline \multicolumn{4}{|l|}{ Duration of RA (years) } \\
\hline Mean \pm SD & $5.5 \pm 9.0$ & $9.0 \pm 9.7$ & $<0.0001$ \\
\hline Median & 1 & 6 & \\
\hline$\% \leq 1$ year & $380(50.7)$ & $7971(22.9 \%)$ & \\
\hline Age at RA onset (years), mean $\pm S D$ & $52.3 \pm 15.4$ & $49.2 \pm 14.8$ & $<0.0001$ \\
\hline Rheumatoid Factor (RF) positivity & $290(69.4 \%)$ & $13,090(69.7 \%)$ & 0.8850 \\
\hline CCP positivity & $154(60.6 \%)$ & $7362(63.4 \%)$ & 0.3620 \\
\hline $\mathrm{CDAI}$, mean $\pm \mathrm{SD}$ & $18.3 \pm 15.0$ & $14.3 \pm 13.1$ & $<0.0001$ \\
\hline \multicolumn{4}{|l|}{ Disease activity } \\
\hline Low & $256(38.4 \%)$ & $16,233(48.7 \%)$ & $<0.0001$ \\
\hline Moderate & $184(27.6 \%)$ & $9559(28.7 \%)$ & \\
\hline High & $227(34.0 \%)$ & $7527(22.6 \%)$ & \\
\hline mHAQ, mean $\pm S D$ & $0.41 \pm 0.48$ & $0.37 \pm 0.46$ & 0.0559 \\
\hline Patient global assessment, mean $\pm S D$ & $36.3 \pm 28.2$ & $31.7 \pm 26.7$ & $<0.0001$ \\
\hline Physician global assessment, mean $\pm \mathrm{SD}$ & $30.8 \pm 23.1$ & $24.8 \pm 21.8$ & $<0.0001$ \\
\hline Patient reported pain, mean $\pm S D$ & $41.8 \pm 28.7$ & $34.3 \pm 27.8$ & $<0.0001$ \\
\hline Patient reported fatigue, mean $\pm S D$ & $35.6 \pm 31.4$ & $38.3 \pm 30.4$ & 0.2509 \\
\hline Reported morning stiffness & $581(82.5 \%)$ & $24,541(73.5 \%)$ & $<0.0001$ \\
\hline$\%$ Duration $\geq 1 \mathrm{~h}$ & $349(49.6 \%)$ & $13,762(41.2 \%)$ & $<0.0001$ \\
\hline NSAID use & $404(87.1 \%)$ & $18,490(84.3 \%)$ & 0.0980 \\
\hline Analgesics use & $375(50.1 \%)$ & $19,381(55.9 \%)$ & 0.0010 \\
\hline
\end{tabular}

$R A$ rheumatoid arthritis, $C C P$ cyclic citrullinated peptide, $C D A I$ Clinical Disease Activity Index, $m H A Q$ Modified health assessment questionnaire, $S D$ standard deviation

${ }^{a} P$ value from Student's two-sample two-sided $t$ test (continuous variables) and Chi-squared test (categorical variables) comparing patients having no identified RA-directed therapy at time of enrollment to those with RA-directed therapies

was more frequent $(82.5 \%$ vs. $73.5 \%)$ and more severe in patients without RA therapy $(49.6 \%$ vs. $41.2 \%$ with stiffness lasting $>1 \mathrm{~h}$ ).

\section{Comorbidities}

Comorbidity histories among the patient subgroups are summarized in Fig. 2. Patients without a history of RA-directed therapy had more frequent occurrences of serious infections prior to enrollment $(59.6 \%$ vs. $51.7 \%$, $P<0.0001)$. However, history of prior malignancy $(6.5 \%$ vs. $9.7 \%, P=0.003)$ and hepatic disease $(1.6 \%$ vs. $3.1 \%, P=0.035)$ were less frequent in the patients without history of RA-directed therapy. 


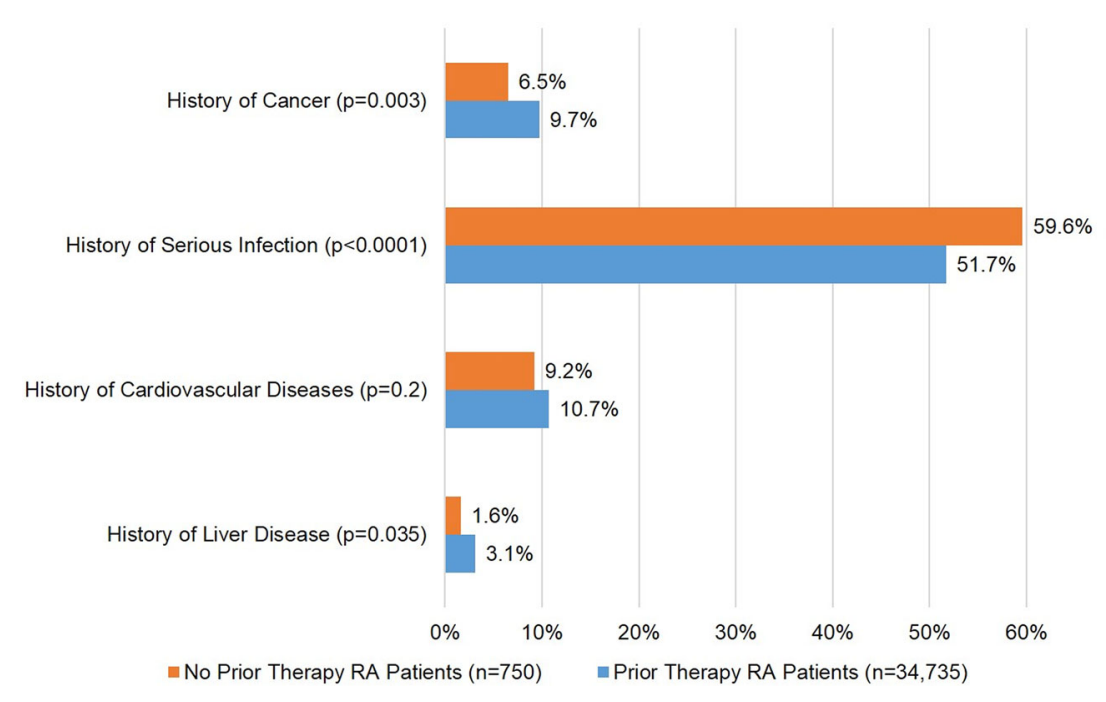

Fig. 2 Comorbidity history of Corrona RA patients at registry enrollment. Cancer excludes non-melanoma skin cancers. Cardiovascular diseases include acute coronary

\section{Time to RA Therapy Initiation}

The group of patients without any history of RA-directed therapy was followed longitudinally for a median (95\% CI) time of 29.5 months (24.6-33.8) since the time of enrollment into Corrona and until the last visit between January 2012 and March 2013. Table 3 summarizes the median months from registry enrollment to initiation of therapy among patients with no history of RA-directed therapy. During the follow-up period, 372 out of $750(49.6 \%)$ patients initiated any RA-directed therapy. The median time to initiation of any RA-directed therapy was 12.1 months (95\% CI: 9.3-14.8).

The Kaplan-Meier survival curves for the initiation of the various RA therapies are shown in Fig. 3. Approximately, $50 \%$ of patients had not started any therapy until 12 months after enrollment to the registry and $25 \%$ of the patients have not started any therapy until 50 months after enrollment in the registry. syndrome, coronary artery disease, congestive heart failure, myocardial infarction, and peripheral arterial disease. $R A$ rheumatoid arthritis

\section{DISCUSSION}

In this descriptive report, we estimated the prevalence of delays in initiation of RA therapy with data from the Corrona registrya large US-based cohort of patients with RA. We estimated the population persistently not receiving RA therapy and calculated the time to initiation of treatment. We described the characteristics of patients not treated promptly and compared them to patients on therapy at the time of enrollment to the Corrona registry.

According to our results, in an era where strong evidence supports the benefits of early initiation of disease-modifying therapy in RA [5], there was still a minority of patients who did not receive appropriate treatment for a long period after diagnosis and after enrollment in the Corrona registry. The percentage of patients not receiving any RA-directed therapy was relatively small $(2.1 \%$ of the total population in the Corrona registry) but the time interval until the initiation of therapy was impressively 
Table 3 Estimated median months from Corrona RA registry enrollment to first drug use among patients with no identified RA-directed therapy at time of enrollment $(N=750)$

\begin{tabular}{llc}
\hline Therapy initiation & $n(\%)$ & Median time in months (95\% CI) \\
\hline $\begin{array}{l}\text { Any drug use } \\
\text { Prednisone, MTX, nbDMARD, or biologic }\end{array}$ & $372(49.6 \%)$ & $12.1(9.3-14.8)$ \\
$\begin{array}{l}\text { First steroid or DMARD use } \\
\text { Prednisone, MTX, or nbDMARD }\end{array}$ & $354(47.2 \%)$ & $14.5(11.3-17.1)$ \\
$\begin{array}{l}\text { First DMARD use excluding steroids } \\
\text { MTX or nbDMARD }\end{array}$ & $325(43.3 \%)$ & $15.9(12.2-18.4)$ \\
\hline
\end{tabular}

long with a median time of 12.1 months to initiation of either steroids, traditional, or biologic DMARDs post-enrollment to the registry. Of note, the cohort of patients on no therapy did not include exclusively those with controlled or relatively inactive RA; half of the patients observed in this analysis had established disease with RA duration of more than 1 year, and more than $60 \%$ of participants had moderate or high disease activity.

Common contraindications to DMARDs, biologic agents, or steroids did not seem to account for such an absence or delay of therapy based on a crude comparison of the prevalence of major comorbidities-such as history of malignancies and cardiovascular diseasebetween patients on therapy and those without. The only exception was the history of serious infections, which were more frequent in subjects without any history of therapy at time of enrollment $(59.6 \%$ vs. $51.7 \%)$. However, while the statistically higher history of serious infections could explain why patients are not treated with biologics or steroids, this should not account for delayed therapy with synthetic DMARDs, which may not be associated with a significant degree of immunosuppression.

Potential factors contributing to the observed delay in therapy could be related to the fact that patients not receiving therapy were less frequently medically insured compared to patients on appropriate therapy. An additional obstacle to initiating therapy could be related to the observed lower level of education in patients not treated appropriately, possibly reflecting different beliefs and health literacy influencing therapeutic decision making [16].

To the best of our knowledge, this is the first study evaluating the frequency and the duration of absence of RA-directed therapy in such a large patient population in the US. Reports show that only a minority of patients are started on appropriate therapy in the first few months upon diagnosis but no reports have dealt with this particular fraction of patients who do not receive therapy for longer periods of time [17-19]. De Cock et al. [13] described a cohort of 156 patients with early RA and identified that only $21.6 \%$ of those patients were started on appropriate DMARD therapy within the first 12 weeks from disease onset. The median time to initiation of appropriate therapy in this study cohort was 23 weeks. Higher disease activity, presence of morning stiffness, and therapy by rheumatologist at an academic center were found to be determinants of earlier initiation of DMARD therapy. Our 

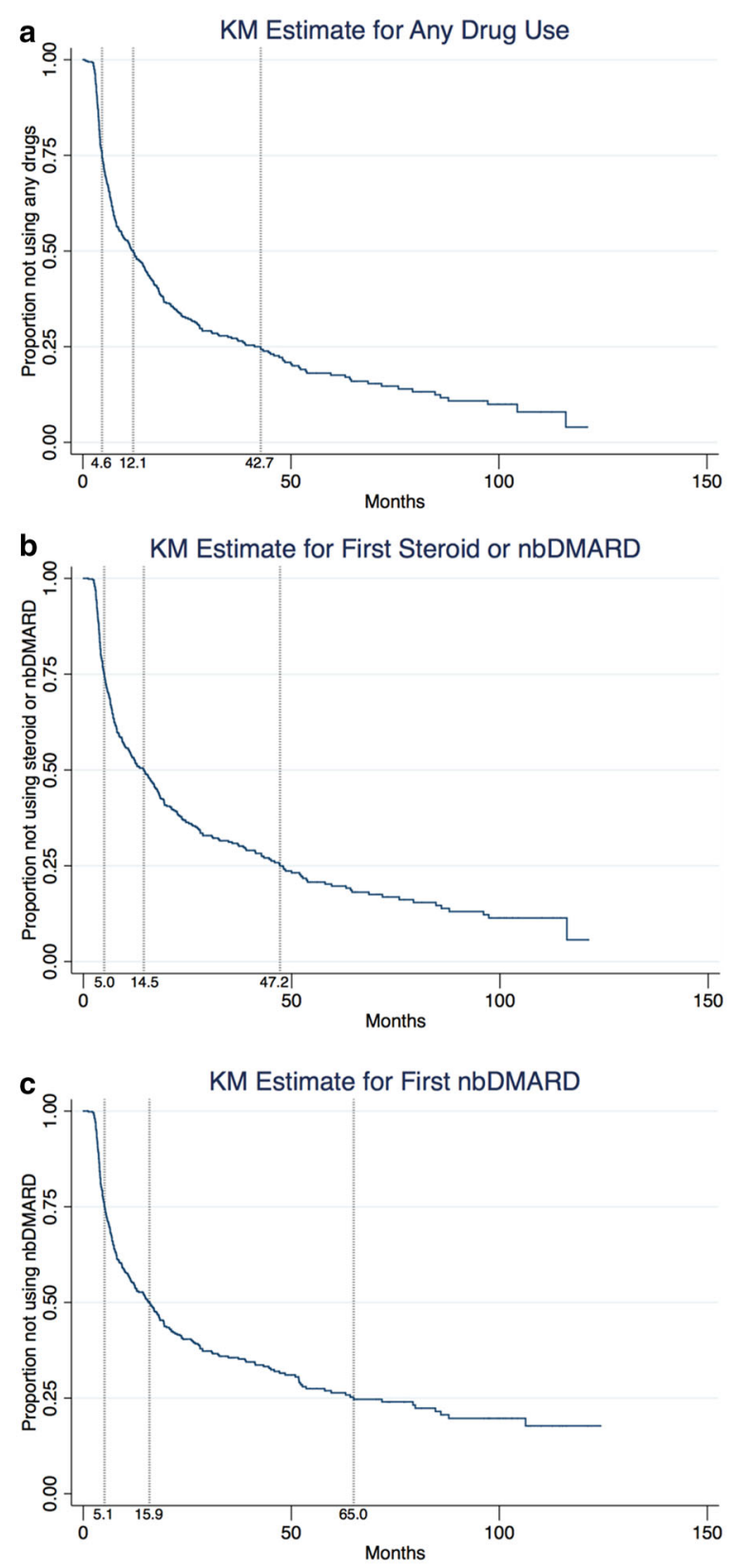

Fig. 3 Estimated survival functions for months from Corrona RA registry enrollment to initiation of a first use of any RA-directed therapy, $\mathbf{b}$ first use of nbDMARDs or steroids, and c first use of nbDMARDs excluding steroids among patients with no history of RA-directed therapy at time of enrollment. $K M$ Kaplan-Meier, $n b D M A R D$ non-biologic disease-modifying antirheumatic drug, $R A$ rheumatoid arthritis study, albeit did not investigate associations between patient or physician characteristics and therapy delays, followed a larger cohort of patients, included early but also established RA patients, and documented much longer delays in initiation of therapy.

Another European study by Potter et al. [14] identified 77 patients with early RA and investigated the reasons for delay in therapy by categorizing them as patient related [delay in presentation to their general practitioner (GP)], GP related, and rheumatologist related. The range of the delay between symptom onset to GP evaluation and then to rheumatologist examination ranged up to several years. Similar results were reported by Kiely et al. [20] where delays in initiation of therapy were due to referrals to secondary care in England. A study from France followed 627 patients with definite and probable RA and reported that 34\% of the patients did not receive DMARDs 6 months after symptom onset; the primary reason for delay was related to uncertainty about diagnosis of RA [21]. Our study also reports long delays but did not aim to attribute the delays observed to specific causes. Our patient population was based in the US with a health system structured in a different way compared to the European systems utilized in the studies mentioned above. In addition, the Corrona registry, although it collects extremely thorough information after enrollment, does not collect pertinent to therapy delays information for the period prior to enrollment to the registry. Nevertheless, our study reports information on a very large US-based patient cohort, followed for a long period of time. 
Our findings have several implications. The US is one of the most developed countries in the world; yet, there is still a fraction of patients with RA who may be facing the spectrum of disability and increased disease activity due to delays in the initiations of therapy. This observation reveals potential barriers and gaps in our health system interfering with appropriate management of a controllable disease. Second, the delay described in this report is not insignificant and places the patients at risk for development of comorbidities associated with uncontrolled disease, decreased quality of life, and increased mortality. It is possible that patients and/or physicians may not be adequately aware of the consequences of untreated RA and educational efforts may need to be pursued to increase awareness of patients and/or physicians.

The strengths of our study are related primarily to the large number of patients enrolled in Corrona and the extended time of follow-up with updated data every 4-6 months post-enrollment. Corrona captures a detailed history of medication usage until the time of enrollment and any initiation or discontinuation of drugs is recorded as they happen during the observation of the patients within the registry over time. In addition, for a multitude of patients, disease and comorbidity related data are reported at enrollment and at each visit, allowing for detailed analyses and associations.

A potential limitation may be that data collected for this analysis began prior to 2014 and before linkage between Corrona and administrative/pharmacy claims databases became available. Thus, it is possible that some patients reported as being on therapy may not be receiving their therapy and vice versa (i.e., some patients reported as not being on therapy may in fact have been on treatment). If the former was the case, linkage with administrative data would strengthen our findings. The latter (i.e., patient misclassified as not receiving therapy) is rather a remote possibility given that medication data are collected in Corrona at multiple time points requiring chart review. The generalizability of our findings remains a potential limitation for this study even though our data are derived from thousands of patients enrolled in 165 rheumatology practices and followed by 625 rheumatologists across all the geographic regions of the US. Furthermore, it is possible that the minority of the patients on no appropriate therapy had some unique characteristics not captured in our registry and rendering them not amenable to therapy (such as compliance issues or beliefs against medication intake). In addition, we did not correlate physician characteristics with delays in therapy. Further, we did not analyze the consequences of delayed therapy on disease outcomes as this has been thoroughly investigated in other studies.

\section{CONCLUSIONS}

In conclusion, we demonstrated that the majority of patients enrolled in the Corrona registry are treated with RA-directed therapy. However, there is still a minority of patients with RA who are not treated with disease-modifying therapy for long periods of time after diagnosis and that these patients did not have lower disease activity than those who were treated. A future analysis using matching methods should identify more detailed disease, patient, and physician factors related to such delays in therapy.

\section{ACKNOWLEDGMENTS}

Corrona, LLC sponsored this study and article processing charges were provided by Horizon 
Pharma. The Corrona RA registry has been supported through contracted subscriptions in the last two years by AbbVie, Amgen, Astra Zeneca, BMS, Genentech, Horizon Pharma USA, Janssen, Eli Lilly, Novartis, Pfizer, and UCB. All named authors meet the International Committee of Medical Journal Editors (ICMJE) criteria for authorship for this manuscript, take responsibility for the integrity of the work as a whole, and have given final approval to the version to be published.

Disclosures. Dimitrios A. Pappas is an employee of Corrona, LLC and a Novartis instructor. Jeffrey D. Kent is an employee of Horizon Pharma USA, Inc. Jeffrey D. Greenberg is an employee and shareholder of Corrona, LLC and has received consulting fees from AstraZeneca, Pfizer, Novartis and Celgene. Marc A. Mason is an employee of Corrona, LLC and holds a joint appointment with the National Institutes of Health. Joel M. Kremer is an employee and shareholder of Corrona, LLC. Robert J. Holt is a consultant for Horizon Pharma USA, Inc.

Compliance with ethics guidelines. All procedures followed were in accordance with the ethical standards of the responsible committee on human experimentation (institutional and national) and with the Helsinki Declaration of 1964, as revised in 2013. Informed consent was obtained from all patients for being included in the study.

Open Access. This article is distributed under the terms of the Creative Commons Attribution-NonCommercial 4.0 International License (http://creativecommons.org/licenses/ by-nc/4.0/), which permits any noncommercial use, distribution, and reproduction in any medium, provided you give appropriate credit to the original author(s) and the source, provide a link to the Creative Commons license, and indicate if changes were made.

\section{REFERENCES}

1. Smolen JS, Aletaha D, Bijlsma JWJ, et al. Treating rheumatoid arthritis to target: recommendations of an international task force. Ann Rheum Dis. 2010;69:631-7.

2. Lard LR, Visser H, Speyer I, et al. Early versus delayed treatment in patients with recent-onset rheumatoid arthritis: comparison of two cohorts who received different treatment strategies. Am J Med. 2001;111:446-51.

3. Van der Heide A, Jacobs JWG, Bijlsma JWJ, et al. The effectiveness of early treatment with 'second-line' antirheumatic drugs: a randomized controlled trial. Ann Intern Med. 1996;124:699-707.

4. Van der Horst-Bruinsma IE, Speyer I, Visser H, et al. Diagnosis and course of early-onset arthritis: results of a special early arthritis clinic compared to routine patient care. Rheumatology. 1998;37:1084-8.

5. Emery P. Evidence supporting the benefit of early intervention in rheumatoid arthritis. J Rheumatol. 2002;66:3-8.

6. Möttönen TT. Prediction of erosiveness and rate of development of new erosions in early rheumatoid arthritis. Ann Rheum Dis. 1988;47:648-53.

7. Van der Heijde DM. Joint erosions and patients with early rheumatoid arthritis. Br J Rheumatol. 1995;34(Suppl 2):74-8.

8. Stenger A. Early effective suppression of inflammation in rheumatoid arthritis reduces radiographic progression. Rheumatology. 1998;37:1157-63.

9. Quinn MA. The therapeutic approach of early intervention for rheumatoid arthritis: what is the evidence? Rheumatology. 2001;40:1211-20.

10. Hochberg MC. Early aggressive DMARD therapy: the key to slowing disease progression in rheumatoid arthritis. Scand J Rheumatol. 1999;28:3-7.

11. Weinblatt ME. Rheumatoid arthritis: treat now, not later! Ann Intern Med. 1996;124:773-4. 
12. Van Nies JAB, Tsonaka R, Gaujoux-Viala C, et al. Evaluating relationships between symptom duration and persistence of rheumatoid arthritis: does a window of opportunity exist? Results on the Leiden Early Arthritis Clinic and ESPOIR cohorts. Ann Rheum Dis. 2015;74(5):806-12.

13. De Cock D, Meyfroidt S, Joly J, et al. A detailed analysis of treatment delay from the onset of symptoms in early rheumatoid arthritis patients. Scand J Rheumatol. 2014;43:1-8.

14. Potter T, Mulherin D, Pugh M. Early intervention with disease-modifying therapy for rheumatoid arthritis: where do the delays occur? Rheumatol. 2002;41:953-5.

15. Kremer JM. The CORRONA database. Autoimmun Rev. 2006;5:46-54.

16. Barton JL, Trupin L, Tonner C, et al. English language proficiency, health literacy, and trust in physician are associated with shared decision making in rheumatoid arthritis. J Rheumatol. 2014;41:1290-7.

17. Chan K-WA, Felson DT, Yood RA, et al. The lag time between onset of symptoms and diagnosis of rheumatoid arthritis. Arthritis Rheum. 1994;37:814-20.
18. Hernandez-Garcia C, Vargas E, Abasolo L, et al. Lag time between onset of symptoms and access to rheumatology care and DMARD therapy in a cohort of patients with rheumatoid arthritis. J Rheumatol. 2000;27:2323-8.

19. Irvine S, Munro R, Porter D. Early referral, diagnosis, and treatment of rheumatoid arthritis: evidence for changing medical practice. Ann Rheum Dis. 1999;58:510-3.

20. Kiely P, Williams R, Walsh D, et al. Contemporary patterns of care and disease activity outcome in early rheumatoid arthritis: the ERAN cohort. Rheumatology. 2009;48:57-60.

21. Benhamou M, Rincheval N, Roy C, et al. The gap between practice and guidelines in the choice of first-line disease modifying antirheumatic drug in early rheumatoid arthritis: results from the ESPOIR cohort. J Rheumatol. 2009;36:934-42. 